ZUMJ-1909-1489

DOI 10.21608/zumj.2019.16613.1489

ORIGINAL ARTICLE

\title{
Atherosclerosis an association with chronic kidney disease in rheumatoid arthritis patients .
}

\author{
Ahmed Abd EL- Moniem Emerah ${ }^{(1)}$, Yasser Abd EL- Hay Mohammad ${ }^{(2)}$, Mohamed \\ Safwat Abd El Dayem ${ }^{(3)}$, Reham Mahmoud Hassan Abdel Fattah ${ }^{(4)}$ \\ (1) Professor of Rheumatology and Rehabilitation, Zagazig University, Egypt \\ (2) Associated Professor of Rheumatology and Rehabilitation, Zagazig University, Egypt. \\ (3) Lecturer of Cardiology, Zagazig University, Egypt \\ (4) MSc student of Rheumatology and Rehabilitation, Zagazig University, Egypt.
}

Corresponding author:

Reham M. Hassan Abdel Fattah; Mobile: 01090464411; Email

address:

drrehammahmoud88@gmail.com

$\begin{array}{ll}\text { Submit Date } & \mathbf{2 0 1 9 - 0 9 - 0 7} \\ \text { Revise Date } & 2019-10-07 \\ \text { Accept Date } & 2019-10-11\end{array}$

ABSTRACT
Background: Rheumatoid arthritis (RA), an autoimmune systemic inflammatory condition, associated with an increased risk of cardiovascular disease (CVD). Recently, renal impairment was suggested to increase the risk of CVD in RA population.

Aim of this study: it was to evaluate the association between impaired renal function and atherosclerosis in patients of rheumatoid arthritis .

Rational: In the light of the increased incidence of renal affection in RA patients and the reported association with the cardiovascular complications which cause excess mortality in patients of rheumatoid arthritis .

We studied if there was association between impaired renal function and accelerating atherosclerosis in rheumatoid arthritis.

Patients and Methods: A cross sectional study on 72 RA patients carried out at Rheumatology and Rehabilitation Department, Faculty of Medicine, Zagazig University. The disease activity was evaluated by the Disease Activity Score (DAS28). Clinical and laboratory assessment was performed with evaluation of carotid intima-media thickness (cIMT) and the presence of atherosclerotic plaques by Carotid ultrasonography, glomerular filtration rate (GFR) was estimated with Modification of diet in renal diseases ( MDRD) formula to define chronic kidney disease (CKD). C-reactive protein (CRP) , rheumatoid factor $(\mathrm{RF})$, erythrocyte sedimentation rate (ESR) and serum amyloid A ( SAA) were used as inflammation markers,

Results: In this study we found that carotid intima-media thickness was abnormal in $33(46 \%)$ of the patients. Age, waist circumference (WC) and disease duration found to be significantly higher in patients with abnormal cIMT. Besides CRP, RF, ESR, SAA and DAS28 were significantly higher with abnormal cIMT, while eGFR was significantly decreased in patients with abnormal cIMT.

In the present study it was found that there was highly negative significant correlation between cIMT and eGFR.

Conclusion: The presence of impaired renal function and long standing inflammation were associated with higher risk of atherosclerosis in RA population.

Keywords: Rheumatoid arthritis, atherosclerosis, CVD risk, impaired renal function, CKD.

\section{INTRODUCTION}

$\mathbf{R}$ A affects $0.5 \%-1 \%$ of adults in developed countries and is three times more common in females than males. Rheumatoid arthritis (RA) is defined by chronic synovitis, systemic inflammation and auto antibodies linked to articular and extraarticular complications [1].

Patients of RA have higher risk of cardiovascular mortality. This high risk is not associated only to the usual cardiovascular risk factors. Rheumatoid arthritis and long standing inflammation associated with it has 
been noted as an independed cardiovascular risk factor [2].

Renal deterioration is popular in rheumatoid arthritis patients. The underline cause of kidney affection in patients of RA is vague, and may be related to nephrotoxic pharmacotherapies, secondary diseases including amyloidosis and/or glomerulonephritis, and co-morbidities associated with the disease [3].

Some studies have shown that deteriorated renal function is linked with increase the risk of cardiovascular diseases in RA [4].

In RA patients there is a significant correlation between disease duration, presence of CVD, high CRP and chronic kidney disease. The association between CVD and CKD remained significant after adjusting for age, gender, duration of arthritis and CRP [5].

The present study was aimed to evaluate the association between impaired renal function and atherosclerosis in patients of rheumatoid arthritis.

\section{1- PATIENTS AND METHODS}

This cross-sectional study was carried out at Rheumatology and Rehabilitation Department, Faculty of Medicine, Zagazig University Hospitals in the period from July 2018 to July 2019. The present study included 72 adult patients with rheumatoid arthritis diagnosed according to The American College of Rheumatology/European League Against Rheumatism collaborative initiative for the classification of RA 2010 [6]. Patients with diabetes mellitus, coronary artery disease, thyroid dysfunction, kidney disease due to other causes such as urinary tract infection and smoking were excluded .

Written informed consent was obtained from all participants and the study was approved by the research ethical committee of Faculty of Medicine, Zagazig University. The work has been carried out in accordance with The Code of Ethics of the World Medical Association (Declaration of Helsinki) for studies involving humans.

Patients were subjected to full history taking and complete examination with stress on age, sex, history of RA including disease duration, extra-articular manifestation and pharmacological therapies used for treatment.
Also traditional cardiovascular risk factors: (hypertension, dyslipidemia, positive family history of CVD, low levels of physical activity and obesity). Anthrobometric assessment was done including weight, height, waist circumference (WC) and body mass index ( BMI) calculation.

Disease activity was measured using the Disease Activity Score (DAS28) based on evaluation of 28 joints [7], calculated with the number of tender joint counts (TJC), swollen joint counts (SJC), erythrocyte sedimentation rate (ESR) value, and general health assessment of the patient which indicated by marking a $10 \mathrm{~cm}$ line between very good and very bad by the patient himself.

Laboratory Tests: Blood was collected for complete blood count (CBC), RF, ESR, CRP levels, fasting blood sugar, serum creatinine, total cholesterol (TC), high density lipoprotein (HDL) cholesterol, low density lipoprotein (LDL) cholesterol, and triglycerides (TG) at the University Hospital lab. Atherogenic index of plasma (AIP) was estimated by logarithm (TG/HDL-C), it is directly related to risk of atherosclerosis[8]. Modification of diet in renal diseases (MDRD) formula was used for every patient to estimate the GFR using serum Creatinine and demographic factors [9] and CKD was defined as an estimated glomerular filtration rate of $<60 \mathrm{~mL} / \mathrm{min} / 1.73 \mathrm{~m} 2$ [10] . SAA was determined by a commercial enzyme-linked immunosorbent assay (ELISA), with detection limit $0.005 \mathrm{mg} / \mathrm{L}$ (Human SAA; SunRED, Shanghi). SAA normal reference range is under $10 \mathrm{mg} / \mathrm{L}$.

Carotid intima-media thickness (cIMT) was measured using high-resolution B-mode ultrasound, Toshiba (diagnostic ultrasound system), model (Aplio Xv), B-mode with pulsed Doppler flow imaging system, using a linear probe with broad band frecuency 614MHZ. IMT was evaluated bilaterally in the three regions: common carotid artery (CCA), carotid bulb, and internal carotid artery (ICA).The average of the maximal IMT from all 6 carotid segments (defined as mean 
cIMT) was used in the analyses. We considered that cIMT $\geq 0.6 \mathrm{~mm}$ is a marker of subclinical atherosclerosis [11]. The presence of carotid plaques is a marker of advanced atherosclerosis. Plaques were defined as a distinct protrusion, greater than $1.5 \mathrm{~mm}$ into the vessel lumen [12].

\section{Statistical analysis}

All data were collected, tabulated and statistically analyzed using SPSS 24.0 for windows (SPSS Inc., Chicago, IL, USA). Data were tested for normal distribution using the Shapiro Walk test. Qualitative data were represented as frequencies and relative percentages. Chi square test $\left(\chi^{2}\right)$ and Fisher exact was used to calculate difference between qualitative variables as indicated. Quantitative data were expressed as mean \pm SD (Standard deviation) for parametric and median and range for non-parametric data. One way ANOVA test was used to compare between more than two dependent groups. Pearson's and Spearman's correlation coefficient were used for correlating normal and non-parametric variables respectively. The (+) sign was considered as indication for direct correlation \& (-) sign as indication for inverse correlation. All statistical comparisons were two tailed with significance Level of Pvalue $\leq 0.05$ indicates significant, $p<0.001$ indicates highly significant difference while, P> 0.05 indicates Non-significant difference.

\section{2- RESULTS}

This study included 17 (23.6\%) males and $55(76.4 \%)$ females with ages ranged from 32 to 57 years old, with a mean BMI of $27.52 \pm 3.48 \mathrm{~kg} / \mathrm{m}^{2}$ and duration of the disease ranged $6-18$ years. Mean cIMT $.787 \pm .263 \mathrm{~mm}$, mean SAA $11.28 \pm 8.21$ $\mu \mathrm{g} / \mathrm{ml}$ and mean eGFR $96.31 \pm 31.13$ $\mathrm{mL} / \mathrm{min} / 1.73 \mathrm{~m}^{2}$.

In the present study it was found that cIMT was abnormal in $33(46 \%)$ of the patients. There was a positive significant correlation between cIMT and age, duration of disease, WC, TG and LDL cholesterol. Also there was a highly positive significant correlation between cIMT and CRP, ESR, RF titer, DAS28 and SAA. There was a highly negative significant correlation between cIMT and HDL cholesterol \& eGFR. [table 2, figure 1].

Table 1: Different parameters among studied patients according to different grades of Disease Activity Score.

\begin{tabular}{|c|c|c|}
\hline Variable & $\begin{array}{l}\text { Mil } \\
\text { Activity }\end{array}$ & $\begin{array}{c}\text { Moderate } \\
\text { Activity }\end{array}$ \\
\hline
\end{tabular}

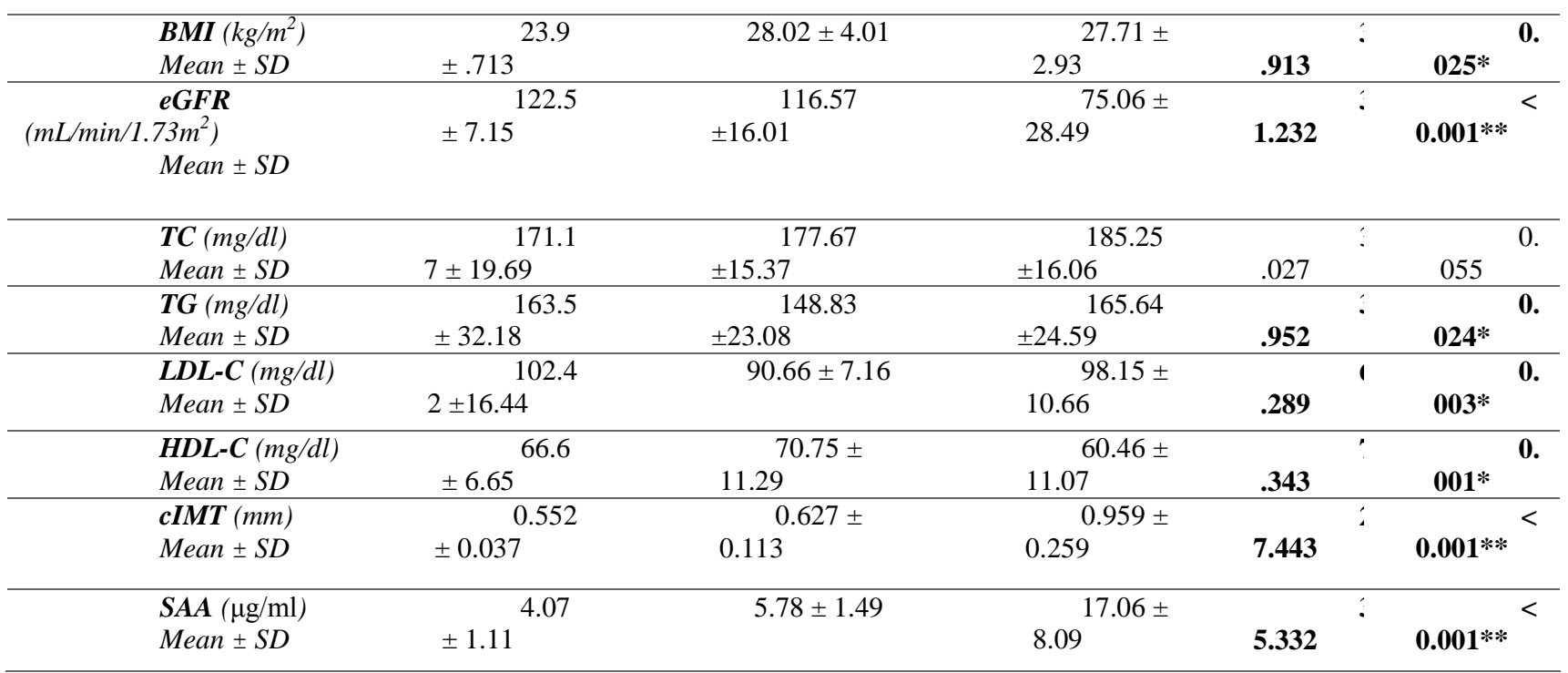

BMI: body mass index, eGFR: estimated glomerular filtration rate, TC: total cholesterol, TG: triglycerides, LDL-C: low-density lipoprotein-cholesterol, HDL-C: high-density lipoproteincholesterol, cIMT: carotid intima media thickness, SAA: Serum amyloid A.

Significant considered $\mathbf{p}<0.05, * *$ Highly Significant considered $\mathbf{p}<0.001$. 
[Table 2]: Correlation of cIMT and SAA with different parameters in all studied patients.

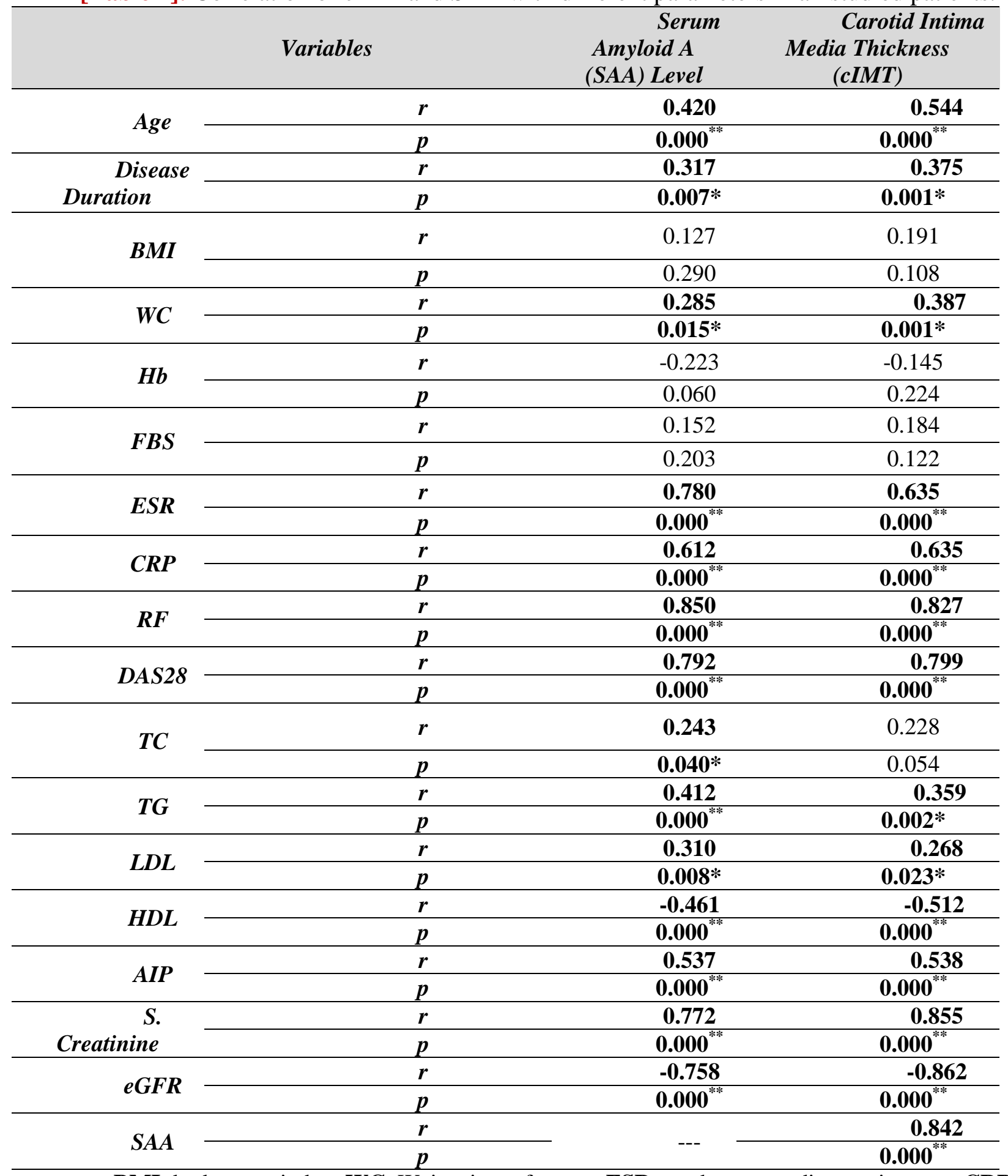

BMI, body mass index. WC, Waist circumference. ESR, erythrocyte sedimentation rate. CRP, Creactive protein. RF, Rheumatoid factor. $\mathbf{E F R}$, estimate the glomerular filtration rate. TC, total cholesterol. TG, triglycerides. LDL-C, low-density lipoprotein-cholesterol. HDL-C, high-density lipoproteincholesterol. DAS28, Disease Activity Score-28. SAA, Serum amyloid A. cIMT, carotid intima media thickness. AIP, Atherogenic index of plasma.

Significant considered $\mathbf{p}<0.05$, ** Highly Significant considered $\mathbf{p}<0.001$. 
Figure (1): Correlation between cIMT and eGFR among the studied RA patients:

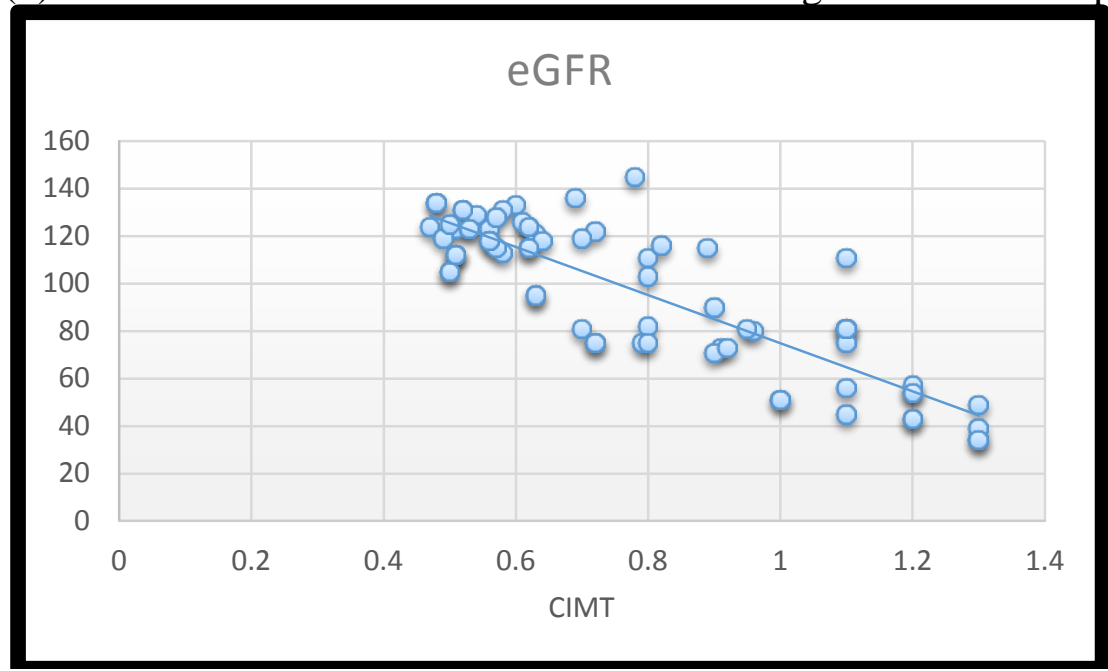

Figure (2) : Correlation between cIMT and estimated SAA.

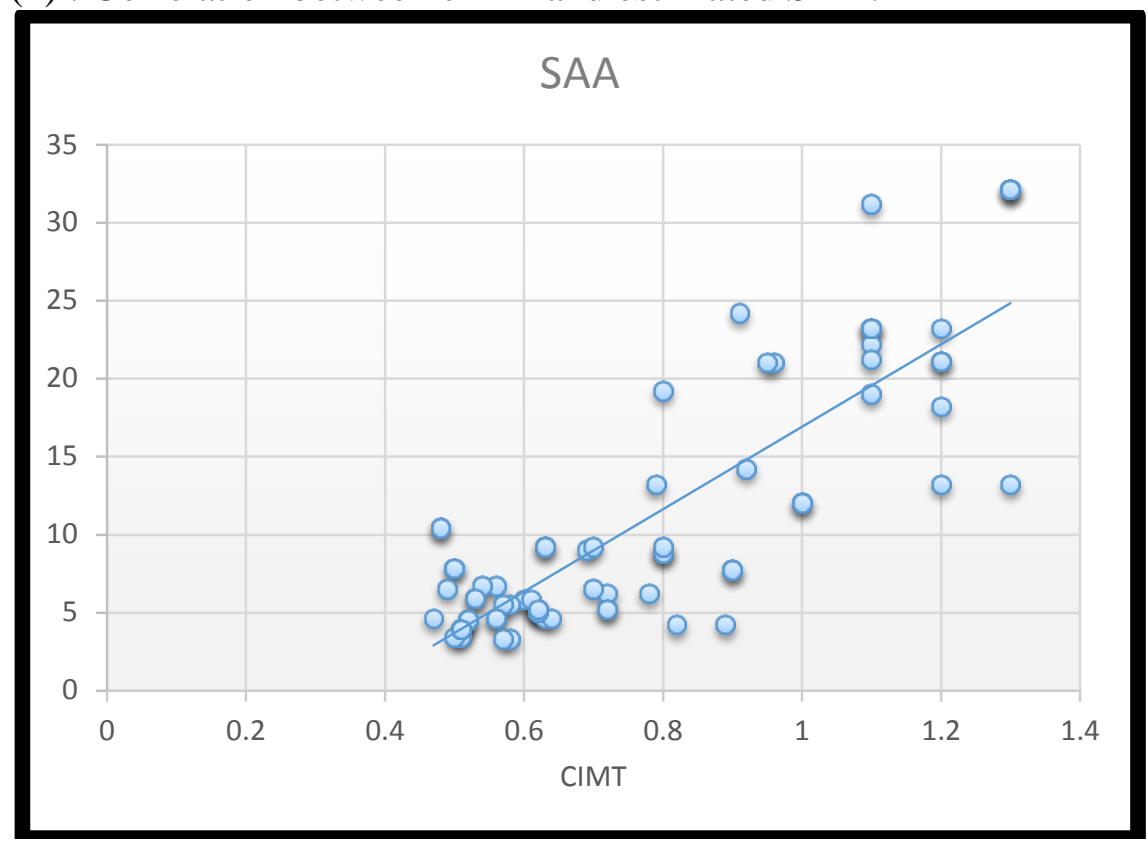

\section{4-DISCUSSION}

Rheumatoid arthritis is an autoimmune disease which cause systemic and articular inflammation that lead to the destructive progression of the joints. Coronary artery disease $(\mathrm{CAD})$ related mortality has been found to be more in RA patients than general population by fifty nine percent [15]. Many studies found a relation between the rheumatoid arthritis, long standing inflammation associated with it and atherosclerosis $[13, \mathbf{1 4}]$. Also it was found that each $5 \mathrm{ml} / \mathrm{min} / 1.73 \mathrm{~m}^{2}$ decrease in GFR cause an increase in the risk of developing a CVD yearly by $11 \%$, independent of traditional risk factors [4].
The aim of this study was to assess the association between impaired renal function and atherosclerosis in rheumatoid arthritis patients.

In the present study; it was found that DAS28 was significantly higher in patients with higher BMI, SAA and abnormal cIMT; [Table 1]. The result was in agreement with Baghdadi et al. [16]. who found that obesity is associated with chronic inflammation and increased levels of circulating adipokines, which might influence the initiation and course of RA. And also agrees with data in literature, which reported a strong correlation between SAA and RA activity [17]. and also with Connolly et al.[18] who documented 
that the SAA level correlated with clinical disease activity. Also agrees with Arts et al.[19] who found that Disease activity over time may contribute to the risk of CVD.

In the present study; it was found that DAS28 was highly significant with lower eGFR and HDL-C; [Table 1]. These results is in agreement with Kochi et al. [20] who concluded that Persistently high disease activity and high CRP was a significant risk factor for the incidence of CKD in RA patients. And also agrees with Georgiadis et al. [21] who found that there was a strong inverse association between disease activity and HDL cholesterol levels in a cohort of patients with early arthritis .

In the present study, it was found that There was a positive significant correlation between cIMT and age, duration of disease and WC [table 2]. These data was in line with Van Sijl et al. [4]. Who reported that RA patients who developed cardiovascular event in his ongoing prospective cohort study were significantly older, with longer disease duration and the incidence was higher in males.

In the present study it was found that there was a positive significant correlation was found between cIMT and age, duration of disease, WC, TG and LDL cholesterol and highly significant correlation with ESR, CRP, RF, DAS28. And there was a highly negative significant correlation between cIMT and HDL [table 2].This result agrees with Hannawi and Alsalmi, [22] who observed that cIMT was positively associated with age of the patients, duration of RA, inflammatory markers such as ESR and CRP, triglyceride level and LDL cholesterol. Besides, cIMT negatively associated with HDL cholesterol. In the contrary, Van Sijl et al.[4] reported no significant difference in ESR, RF and DAS28 between RA patients with and without CV events. And this is may be explained by the differences in inclusion and exclusion criteria.

Chronic kidney disease, known as an independent risk factor for CVD in the general population, is a frequent comorbidity among RA patients and was recently suggested to increase the risk of CVD in this population [ 23]. In the present study, it was found that there was highly negative significant correlation between cIMT and eGFR [table 2, figure 1]. This results in agreement with Van Sijl et al. [4] who noted that patients who had a CV event had substantially and significantly higher serum levels of creatinine and lower GFR than patients who did not have a CV event.

In the present study, it was found that there was highly positive significant correlation between SAA level and cIMT [table 2, Figure 2]. This result agrees with TargoNska-Stwpniak and Majdan [24], who found that there was a relationship between SAA and CV risk factors. The mean SAA level was significantly higher in patients with plaques as a manifestation of advanced atherosclerosis. However, Jylhävä et al.[25] reported no independent effect of SAA on cIMT value, unlike our results, This difference may be explained by that in our study we excluded diabetic and metabolic risk factors meanwhile Jylhävä et al [25]. studied many parameters of early atherosclerosis, i.e. carotid artery compliance and intima- media thickness, as well as serum lipids, proteins and hormones, obesity indices, smoking habits, blood pressure values, alcohol consumption, physical activity, the presence of diabetes and rheumatic diseases.

In the present study, a positive significant correlation was found between SAA and disease duration and a highly positive significant correlation with age, ESR, CRP, RF and DAS28 [table 2]. This result agrees with Shen et al. [26] who found a significant positive correlation between SAA and ESR, CRP as well as disease activity in patients of RA.

In the present study, there was highly negative significant correlation between SAA and eGFR as an early marker of chronic kidney disease (CKD) [table 2]. This result is in agreement with TargoNska-Stwpniak and Majdan. [24] study which indicated that, in RA patients SAA could be considered as a biomarker associated with the inflammatory disease activity and the chronic complications related to the course of RA as the risk of $\mathrm{CV}$ and renal impairment .

\section{5- CONCLUSION}

This study evaluated the relation between chronic impaired renal function and 
atherosclerosis and confirmed the highly significant negative correlation between impaired renal function and atherosclerosis.

Also this study evaluated Serum Amyloid A level and its relation with increasing the risk of cardiovascular and renal affection. And SAA level was found to be in negatively significant correlation with eGFR, and in a highly positive significant correlation with disease activity, other inflammatory markers and cIMT. This suggests that Serum Amyloid A can be used as a marker of persistent inflammation and an indicator of cardiovascular and renal involvement in patients with rheumatoid arthritis.

\section{6- RECOMMENDATIONS:}

- Controlling RA flare ups will help in decreasing the risk of Atherosclerosis progression.

- Routine follow up of lipid profile and weight to limit risk factors of dyslipidemia and Atherosclerosis.

- Estimation of the atherogenic index of plasma as it is directly related to risk of atherosclerosis.

- Monitoring of renal function as chronic renal dysfunction increase the risk of atherosclerosis.

- Cautious usage of nephrotoxic drugs in RA patients with regular monitoring of the kidney function.

- Monitoring of SAA level to asses disease activity in RA patients, Also as it can be considered as a predictor of Atherosclerosis and renal involvement.

\section{7- LIMITATIONS:}

- The sample size was not too large.

- Few patients were not well cooperative in doing some laboratory tests.

Acknowledgements

We thank all the clinicians and patients for their cooperation in the study.

\section{Conflicts of interest:}

The authors declare that they have no competing interests.

\section{8- REFERENCES}

[1] Choy E. Understanding the dynamics: pathways involved in the pathogenesis of rheumatoid arthritis. Rheumatology. $2012 \mathrm{Jul}$ 1;51(5):v3-v11.
[2] Carbone F, Bonaventura A, Liberale L, Paolino S, Torre F, Dallegri F, et al. Atherosclerosis in rheumatoid arthritis: promoters and opponents. Clinical reviews in allergy \& immunology. 2018 Sep 26:1-4.

[3] Couderc M, Tatar Z, Pereira B, Tiple A, Gilson M, Fautrel B, et al. Prevalence of Renal Impairment in Patients With Rheumatoid Arthritis: Results From a Cross- Sectional Multicenter Study. Arthritis care \& research. 2016 May;68(5):638-44.

[4] Van Sijl AM, Van den Oever IA, Peters MJ, Boers M, Dijkmans BA, Van Halm VP, et al. Subclinical renal dysfunction is independently associated with cardiovascular events in rheumatoid arthritis: the CARRÉ Study. Annals of the rheumatic diseases. 2012 Mar 1;71(3):341-44.

[5] Haroon M, Adeeb F, Devlin J, Gradaigh D and Walker F. A comparative study of renal dysfunction in patients with inflammatory arthropathies: strong association with cardiovascular diseases and not with antirheumatic therapies, inflammatory markers or duration of arthritis. Int $\mathrm{J}$ Rheum Dis.2011; 14: 255-60.

[6] Aletaha D, Neogi T, Silman AJ, Funovits J, Felson DT, Bingham CO 3rd, et al. 2010 rheumatoid arthritis classification criteria: an American College of Rheumatology/European League Against Rheumatism collaborative initiative. Arthritis \& Rheumatism. 2010 Sep;62(9):2569-81.

[7] Van Gestel A, Haagsma C and Van Riel PL. Validation of rheumatoid arthritis improvement criteria that include simplified joint counts. Arthritis \& Rheumatism: Official Journal of the American College of Rheumatology. 1998 Oct;41(10):1845-50.

[8] Niroumand S, Khajedaluee M, KhademRezaiyan M, Abrishami M, Juya M, Khodaee G, et al. Atherogenic Index of Plasma (AI): A marker of cardiovascular disease. Medical journal of the Islamic Republic of Iran. 2015; 29: 240.

[9] Levey AS, Bosch JP, Lewis JB, Greene T, Rogers N and Roth D. A more accurate method to estimate glomerular filtration rate from serum creatinine: a new prediction equation. Annals of internal medicine. 1999 Mar 16;130(6):461-70.

[10] Levey, Coresh J, Balk E, Kausz AT, Levin A, Steffes MW, et al. "National Kidney Foundation practice guidelines for chronic kidney disease: evaluation, classification, and 
stratification." Annals of internal medicine. 2003; 139 (2): 137-47.

[11] Randrianarisoa E, Rietig R, Jacob S, Blumenstock G, Haering HU, Rittig K, et al. Normal values for intima-media thickness of the common carotid artery--an update following a novel risk factor profiling. Vasa. 2015 Nov 1;44(6):444-50.

[12] Gonzalez-Juanatey C, Llorca J, Martin J and Gonzalez-Gay MA. Carotid intimamedia thickness predicts the development of cardiovascular events in patients with rheumatoid arthritis. InSeminars in arthritis and rheumatism 2009 Apr 1 ;38(5): 366-71.

[13] Cavagna L, Boffini N, Cagnotto G, Inverardi $\mathbf{F}$, Grosso $\mathbf{V}$ and Caporali $\mathbf{R}$. Atherosclerosis and rheumatoid arthritis: more than a simple association. Mediators of inflammation. 2012; Article ID 147354.

[14] Urman A, Taklalsingh N, Sorrento $C$ and McFarlane IM. Inflammation beyond the Joints: Rheumatoid Arthritis and Cardiovascular Disease. SciFed journal of cardiology. 2018;2(3).

[15] Mohan A, Sada S, Kumar BS, Sarma KV, Devi BV, Rao PV, et al. Subclinical atherosclerosis in patients with rheumatoid arthritis by utilizing carotid intima-media thickness as a surrogate marker. The Indian journal of medical research. 2014 Sep;140(3):379.

[16] Baghdadi LR, Woodman RJ, Shanahan EM, Mangoni AA. The Impact of Traditional Cardiovascular Risk Factors on Cardiovascular Outcomes in Patients with Rheumatoid Arthritis: A Systematic Review and Meta-Analysis.2015; PLoSONE10 (2): $\mathrm{e} 0117952$.

[17] Cunnane G, Grehan S, Geoghegan S, McCormack C, Shields D, Whitehead AS, $\boldsymbol{e t}$ al. Serum amyloid $\mathrm{A}$ in the assessment of early inflammatory arthritis. $\mathrm{J}$ Rheumatol.2000;27(1):58-63.

[18] Connolly M, Mullan RH, McCormick J, Matthews C, Sullivan O, Kennedy A, et al. Acute-phase serum amyloid A regulates tumor necrosis factor alpha and matrix turnover and predicts disease progression in patients with inflammatory arthritis before and after biologic therapy. Arthritis Rheum.2012; 64(4):1035-45.

[19] Arts EE, Fransen J, den Broeder AA, Popa $\mathrm{CD}$, van Riel PL. The effect of disease duration and disease activity on the risk of cardiovascular disease in rheumatoid arthritis patients. Annals of the rheumatic diseases. 2015; 74(6), 998-1003.

[20] Kochi M, Kohagura K, Shiohira Y, Iseki K, Ohya Y. Inflammation as a risk of developing chronic kidney disease in rheumatoid arthritis. PLoS One. 2016; 11(8): e0160225.

[21] Georgiadis AN, Papavasiliou EC, Lourida ES, Alamanos Y, Kostara C, Tselepis AD, $\boldsymbol{e t}$ al. Atherogenic lipid profile is a feature characteristic of patients with early rheumatoid arthritis: effect of early treatment, a prospective, controlled study. Arthritis Res. Ther.2006; 8(3): R82.

[22] Hannawi S and Alsalmi I . Renal function contribute to risk of cardiovascular disease in rheumatoid arthritis. Annals of the Rheumatic Diseases. 2017;76:1043-44.

[23] Kronbichler A and Mayer G . Renal involvement in autoimmune connective tissue diseases. BMC Med.2013; 11: 95.

[24] Targońska-Stępniak $B$ and Majdan $M$. Serum amyloid A as a marker of persistent inflammation and an indicator of cardiovascular and renal involvement in patients with rheumatoid arthritis. Mediators of inflammation. 2014; Article ID: 793628.

[25] Jylhävä J, Haarala A, Eklund C, Pertovaara M, Kähönen M, Hutri- Kähönen $\mathbf{N}$, et al. Serum amyloid A is independently associated with metabolic risk factors but not with early atherosclerosis: the Cardiovascular Risk in Young Finns Study. J Intern Med.2009; 266(3):286-95.

[26] Shen C, Sun X, Liu N, Mu Y, Hong C, Wei $\mathbf{W}$, et al. Increased serum amyloid A and its association with autoantibodies, acute phase reactants and disease activity in patients with rheumatoid arthritis. Molecular medicine reports. $2015 \mathrm{Feb}$ 1;11(2):1528-34.

How to Cite

hassan, R., Emerah, A., Mohammad, Y., Abd El Dayem, M. Atherosclerosis an Association with Chronic Kidney Disease in Rheumatoid Arthritis Patients. Zagazig University Medical Journal, 2021; (477-484): -. doi: 10.21608/zumj.2019.16613.1489 